University of Nebraska - Lincoln

DigitalCommons@University of Nebraska - Lincoln

US Department of Energy Publications

U.S. Department of Energy

2012

\title{
Field ion source development for neutron generators
}

B. Bargsten Johnson

University of New Mexico, Albuquerque, NM

P. R. Schwoebel

University of New Mexico, Albuquerque, NM

C. E. Holland

SRI International, Menlo Park,CA

P. J. Resnick

Sandia National Laboratories, Albuquerque, NM

K L. Hertz

Sandia National Laboratories, Livermore, CA

See next page for additional authors

Follow this and additional works at: https://digitalcommons.unl.edu/usdoepub

Part of the Bioresource and Agricultural Engineering Commons

Johnson, B. Bargsten; Schwoebel, P. R.; Holland, C. E.; Resnick, P. J.; Hertz, K L.; and Chichester, D L., "Field ion source development for neutron generators" (2012). US Department of Energy Publications. 120.

https://digitalcommons.unl.edu/usdoepub/120

This Article is brought to you for free and open access by the U.S. Department of Energy at DigitalCommons@University of Nebraska - Lincoln. It has been accepted for inclusion in US Department of Energy Publications by an authorized administrator of DigitalCommons@University of Nebraska - Lincoln. 


\section{Authors}

B. Bargsten Johnson, P. R. Schwoebel, C. E. Holland, P. J. Resnick, K L. Hertz, and D L. Chichester 


\title{
Field ion source development for neutron generators
}

\author{
B. Bargsten Johnson ${ }^{\text {a }}$, P.R. Schwoebel ${ }^{\mathrm{a}, *}$, C.E. Holland ${ }^{\mathrm{b}}$, P.J. Resnick ${ }^{\mathrm{c}}$, K.L. Hertz ${ }^{\mathrm{d}}$, D.L. Chichester $^{\mathrm{e}}$ \\ a University of New Mexico, Albuquerque, NM 87131, USA \\ b SRI International, Menlo Park, CA 94025, USA \\ c Sandia National Laboratories, Albuquerque, NM 87123, USA \\ ' Sandia National Laboratories, Livermore, CA 94551, USA \\ e Idaho National Laboratory, Idaho Falls, ID 83415, USA
}

\section{A R T I C L E I N F O}

\section{Article history:}

Received 11 July 2011

Received in revised form

8 September 2011

Accepted 15 September 2011

Available online 28 September 2011

\section{Keywords:}

Field desorption

Field ionization

Neutron generator

Ion source

Deuterium

Tritium

\begin{abstract}
A B S T R A C T
An ion source based on the principles of electrostatic field desorption is being developed to improve the performance of existing compact neutron generators. The ion source is an array of gated metal tips derived from field electron emitter array microfabrication technology. A comprehensive summary of development and experimental activities is presented. Many structural modifications to the arrays have been incorporated to achieve higher tip operating fields, while lowering fields at the gate electrode to prevent gate field electron emission which initiates electrical breakdown in the array. The latest focus of fabrication activities has been on rounding the gate electrode edge and surrounding the gate electrode with dielectric material. Array testing results have indicated a steady progression of increased array tip operating fields with each new design tested. The latest arrays have consistently achieved fields beyond those required for the onset of deuterium desorption $(\sim 20 \mathrm{~V} / \mathrm{nm})$, and have demonstrated the desorption of deuterium at fields up to $36 \mathrm{~V} / \mathrm{nm}$. The number of ions desorbed from an array has been quantified, and field desorption of metal tip substrate material from array tips has been observed for the first time. Gas-phase field ionization studies with $\sim 10,000$ tip arrays have achieved deuterium ion currents of $\sim 50 \mathrm{nA}$. Neutron production by field ionization has yielded $\sim 10^{2} \mathrm{n} / \mathrm{s}$ from $\sim 1 \mathrm{~mm}^{2}$ of array area using the deuterium-deuterium fusion reaction at $90 \mathrm{kV}$.
\end{abstract}

(c) 2011 Elsevier B.V. All rights reserved.

\section{Introduction}

Terrorism and nuclear proliferation concerns have made the detection of nuclear weapons, improvised nuclear devices, radiological dispersal devices, and their key components a major focus of homeland and national security activities [1]. The detection and identification of special nuclear materials (SNM), in particular highly enriched uranium (HEU), is a high priority. A combination of factors such as the small amounts required for an explosive device, the large quantities in the world inventory, and difficulty of detecting HEU by passive radiation monitoring make securing and controlling HEU one of the greatest global security challenges of the current era.

Passive monitoring systems cannot reliably address the most challenging problems involving detection and identification of shielded HEU. For these cases active interrogation techniques such as neutron interrogation are an effective and, in some instances, the only reliable means to remotely detect such nuclear materials [2]. A National Academy of Sciences study has

\footnotetext{
* Corresponding author. Tel.: +1 5052778488.

E-mail address: schwoebel@chtm.unm.edu (P.R. Schwoebel).
}

concluded that a critical component hindering the development of fieldable active neutron interrogation systems is the neutron generator [3]. Existing compact neutron generators [4,5] do not meet the combined output, size, weight, lifetime, and power requirements of field detection systems.

To improve the performance of existing compact neutron generators, an ion source capable of producing deuterium or mixed species deuterium-tritium ion beams based on principles of electrostatic field desorption (EFD) is being investigated [6]. Field desorption uses high electric fields $(20-40 \mathrm{~V} / \mathrm{nm})$ to remove, as ions, atomic deuterium and/or tritium adsorbed from the gas phase onto a surface. Desorption is initiated from the surfaces of metal tips such that the high electric fields required can be achieved using modest applied voltages. The ions produced from this source are used to drive the standard deuterium-deuterium (D-D) or deuterium-tritium (D-T) fusion reactions to generate neutrons.

In order to achieve the ion currents required for an intense neutron source, an array of desorption tips is required. The basic microstructure for the field desorption ion source array is derived from field emitter array (FEA) microfabrication technology [7]. The ion source array structure is shown schematically in Fig. 1, and has been described in detail previously [8]. 


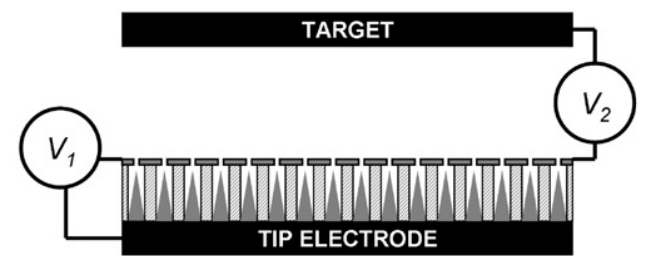

Fig. 1. Schematic of the field desorption array ion source as a D-D (or D-T) neutron generator. $V_{1}$ applied between the base and gate electrodes generates deuterium ions independent of the accelerating voltage, $V_{2}$.

The key operational feature is the use of a relatively low voltage pulse, $V_{1}(\sim 1 \mathrm{kV})$, applied between micron-spaced gate and tip electrodes to generate a field at the tip apices sufficient for deuterium/tritium desorption ( $>20 \mathrm{~V} / \mathrm{nm}$ ). $V_{1}$ is independent of the potential, $V_{2}(\sim 100 \mathrm{kV})$, used to accelerate the ions toward the target. The array structure operates in atmosphere of deuterium or deuterium/tritium gas so molecules can re-adsorb on the tip surfaces between voltage pulses.

The field desorption arrays are capable of operating at both room and liquid nitrogen temperature. It has been shown that the maximal fields for desorption from molybdenum and tungsten without removing tip material are $\sim 35$ and $\sim 44 \mathrm{~V} / \mathrm{nm}$, respectively [9]. This magnitude of the electric field is sufficient to remove a large fraction of the adsorbed atoms present on the tip surfaces in less than a few nanoseconds. The resulting short neutron pulse allows the use of coincidence techniques during active interrogation.

The array structures are also capable of achieving fields required for a related, but fundamentally different process, field ionization [10]. Field ionization provides an alternative ion source operating mode with applied d.c. voltages rather than pulsed voltages. The onset of field ionization with hydrogen isotopes occurs at $\sim 10 \mathrm{~V} / \mathrm{nm}$, lower fields than those required for desorption. Using field ionization, a d.c. voltage $\left(V_{1}\right.$ in Fig. 1$)$ is applied, and the resulting electric field ionizes a region of gas in front of the tip apices to generate a hydrogen isotope ion current.

\section{Experimental apparatus and procedure}

The experimental apparatuses used for studying field desorption and field ionization ion sources were ion-pumped, stainless steel ultrahigh vacuum chambers operating with base pressures of $\sim 10^{-10}$ Torr following a 10 hour bake-out at $\sim 200{ }^{\circ} \mathrm{C}$. Two different chamber configurations were used: 1) An imaging atom probe for traditional time-of-flight (TOF) mass analysis, and 2) a light ion accelerator for neutron production. For both configurations, a glass cold finger served as the mechanical and electrical mount for the arrays. The cold finger can be filled with liquid nitrogen for operation at $\sim 77 \mathrm{~K}$. Research grade purity (99.9999\%) deuterium gas is admitted to the vacuum system through controllable leak valves from one liter glass flasks. Note that for these studies, $77 \mathrm{~K}$ refers to the operating temperature of the array, and not that of the deuterium gas, which is at approximately room temperature. Due to the practical difficulties of using tritium in vacuum pumped systems, our research activities to-date have exclusively used deuterium gas; tritium gas will be used in future work when efforts shift to developing sealed-tube neutron generators.

\subsection{Time-of-flight arrangement}

Fig. 2 shows the experimental arrangement for the imaging atom probe configuration in which the target in Fig. 1 has been

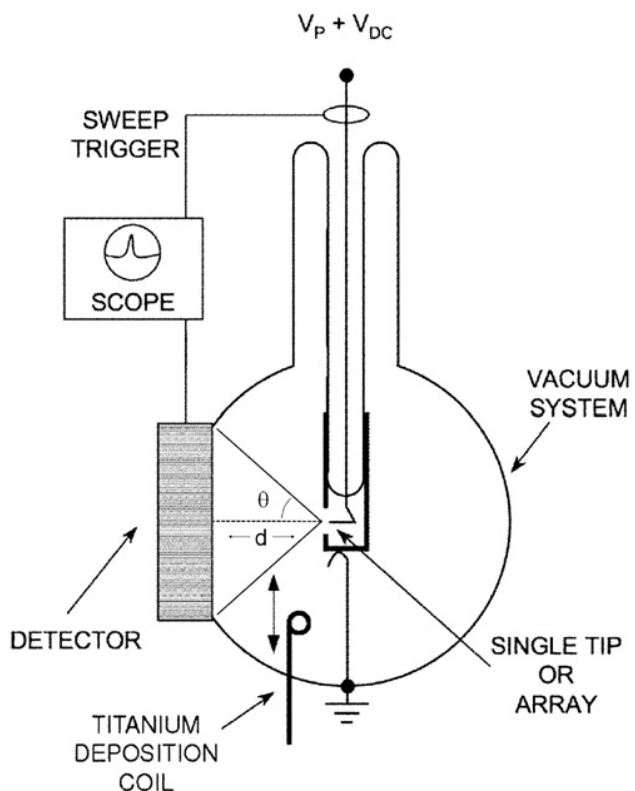

Fig. 2. Schematic of the ultrahigh vacuum atom-probe. The half-angle, $\theta$, defines the emission cone from a single wire tip or an array. $d$ is the distance from the array or tip to the detector.

replaced with a chevron type channel electron multiplier array (CEMA) ion detector [8]. The CEMA output is projected onto a P-47 phosphor screen and the signal viewed with a photomultiplier tube. $24.8 \mathrm{~mm}$ and $86.7 \mathrm{~mm}$ CEMAs were used, with the array-to-detector distance, $d$, varying between $7 \mathrm{~cm}, 10 \mathrm{~cm}$, or $13 \mathrm{~cm}$ depending upon the vacuum chamber. The half-angle, $\theta$, represents the emission cone for a given array or single etchedwire tip.

To initiate field desorption for the TOF experiments, voltage pulses were applied to the tip electrode, relative to a grounded gate electrode. Peak voltage on the order of $1 \mathrm{kV}$ in the form of 2 ns rise-time, 20 ns duration pulses were applied at a frequency of $1 \mathrm{~Hz}$ using a custom built cable discharge-type pulse generator. The leading edges of the TOF peaks are used to identify mass species in the TOF spectra [11].

Due to the inherent capacitance of the array, there is a slight impedance mismatch with the $50 \Omega$ impedance of the pulse drive circuit. The resulting negative going pulse reflections, which are typically $20 \%$ of the applied voltage pulse magnitude, can be sufficient to induce field electron emission from the tips. To prevent electron emission, the desorption voltage pulse is coupled onto a positive d.c. holding voltage. This d.c. voltage level is chosen so that the resulting applied d.c. electric field at the tips remains below the onset of field ionization $(\sim 10 \mathrm{~V} / \mathrm{nm})$.

\subsection{Field ionization and field electron measurements}

The imaging atom probe configuration is used to directly measure both gas-phase field ion (deuterium) current and field electron emission current from arrays incident on the CEMA. Gasphase field ionization currents were produced using a d.c. voltage applied to the tips while the gate was held at ground and measured with a Keithley 486 picoammeter. Field electron emission current was generated with a negative $60 \mathrm{~Hz}$ half-wave rectified sinusoidal a.c. voltage applied to the tips with the gate grounded and measured on a Tektronix 7603 oscilloscope. In this case CEMA is biased to $+300 \mathrm{~V}$.

Due to the trade-off between mass resolution and image area in the imaging atom probe [11], the CEMA may not collect all ions emitted from the array for a given array-to-detector distance. 
Corrections for this effect were calculated and accounted for in the data where appropriate. For field electron emission measurements and neutron production experiments all charged species are collected due to bias on the collector and target, respectively.

Field electron emission and gas-phase ionization currents are used to calculate and calibrate the fields on the tip apices of an array. The field, $F_{1}$, is determined from the voltage-field proportionality relation $V_{1} / V_{c}=F_{1} / F_{c}$ where $V_{1}$ is voltage applied to the array. The calibration voltages and fields, $V_{c}$ and $F_{c}$, are determined for the onsets of field electron emission and deuterium field ionization, respectively. For field electron emission, $V_{c}$ is the voltage required to produce $0.1 \mathrm{nA} /$ tip of electron current from an array at an assumed field, $F_{c}$, of $2.5 \mathrm{~V} / \mathrm{nm}$. For deuterium field ionization, $V_{c}$, is the voltage required to produce $0.01 \mathrm{pA} /$ tip of ion current from an array at an assumed field, $F_{c}$, of $10 \mathrm{~V} / \mathrm{nm}$ (at $77 \mathrm{~K}$ ).

\subsection{Neutron production measurements}

A light ion accelerator configuration was used for neutron production experiments with arrays operating in the field ionization mode at $77 \mathrm{~K}$. For this system, the target in Fig. 1 is a $\mathrm{TiD}_{2}$ film on a copper disk [12]. The ion accelerating voltage, $V_{2}$, was $-90 \mathrm{kV}$ applied across a $10 \mathrm{~cm}$ array-to-target gap.

The $\sim 2.5 \mathrm{MeV}$ neutrons produced were detected with a custom-built, ${ }^{3} \mathrm{He}$ detector [13]. The active detection region containing the ${ }^{3} \mathrm{He}$ tubes is $30 \mathrm{~cm} \times 55 \mathrm{~cm} \times 10 \mathrm{~cm}$. Thermal background neutrons are shielded by a $0.38 \mathrm{~mm}$ thick layer of cadmium integrated into the detector. Additional shielding was utilized to surround five sides with $14 \mathrm{~cm}$ of paraffin (Texwax). The sixth side of the detector was centered on the $10 \mathrm{~cm}$ distant $\mathrm{TiD}_{2}$ target, subtending a solid angle of 3.7 sr. Modeling and calibration with an AmBe source had previously determined the intrinsic detector efficiency to be $20 \%$. Each of the four output channels of the ${ }^{3} \mathrm{He}$ detector were fed to analog counters (Ortec 771 , Ortec $772\{\times 2\}$, Tenelec TC 540 A). Typical neutron counts were conducted over $60 \mathrm{~s}$ time intervals with background subtraction. For reference $\sim 1 \mathrm{nA}$ of field ion current was sufficient to distinguish the neutron signal from background by 3-sigma.

\subsection{Single etched-wire tip calibration of deuterium ion yields}

Characterization of ion emission from single etched-wire tips provided basic understanding of the expected performance of the arrays. For these experiments, an extruded tungsten wire was etched in a sodium hydroxide solution [9] to create a tip radius of $\sim 20 \mathrm{~nm}$, similar to that of the array tips. Desorption experiments and titanium film evaporation experiments were performed to quantify the amount of deuterium ions desorbed using a technique described previously $[14,15]$. Titanium films were formed on the tip surface by in situ deposition from a titanium getter-wire mounted on a linear feed through (see Fig. 2).

For our $\left\langle\begin{array}{lll}1 & 1 & 0\end{array}\right\rangle$ oriented etched-wire tungsten tip, the tip radius, $r_{\text {tip }}$, was calculated using the relation $F=V /\left(5 r_{\text {tip }}\right)$ [16]. Here $V$ was the voltage applied to the tip at the best imaging fields, $F$, for $\mathrm{D}_{2}$ and He gas; taken to be $22 \mathrm{~V} / \mathrm{nm}$ and $44 \mathrm{~V} / \mathrm{nm}$ at $77 \mathrm{~K}$, respectively [17].

\section{Discussion and results}

Array designs were driven by modeling results, which focused on maximizing the tip fields while keeping gate electrode fields below field electron emission thresholds. Early modeling results have been discussed [18], and the modeling results leading to the present designs will be presented in a future publication [19].
The gated array design has two major advantages over a diode approach consisting of ungated array tips and a target electrode. First, more tips per unit source area can be achieved with a gate electrode due to the lack of mutual shielding between tips. This increases the output of the array as the ion yield is proportional to the number of tips. Second, with a gated electrode, pulsed operation requires applied voltages on the order of kilovolts, and not tens of kilovolts, as with diode operation. This greatly reduces the weight and power burdens of the pulsing supply which is advantageous for field portable interrogation systems.

\subsection{Array fabrication evolution}

Two different microfabrication approaches are utilized to fabricate array devices. SRI International in Menlo Park, Calif. uses an approach based on the fabrication of Spindt field emission array (FEA) cathodes while the Microsystems and Engineering Sciences Applications facility at Sandia National Laboratories (SNL) in Albuquerque, N.M. uses MEMS surface micromachining. The different design iterations developed are shown in Fig. 3a-h, and are discussed in chronological order. Here, the array geometries are described as they are correlated with the experimental results, whereas details of the fabrication processes have been [20], or will be, published elsewhere.

\subsubsection{SRI fabrication}

Fig. 3a is a schematic of a standard FEA structure. A silicon wafer serves as the base electrode and substrate on which molybdenum tips are grown. The chromium gate electrode is separated by a $\sim 0.5 \mu \mathrm{m}$ layer of silicon dioxide, $\mathrm{SiO}_{2}$. Holes of submicron diameter

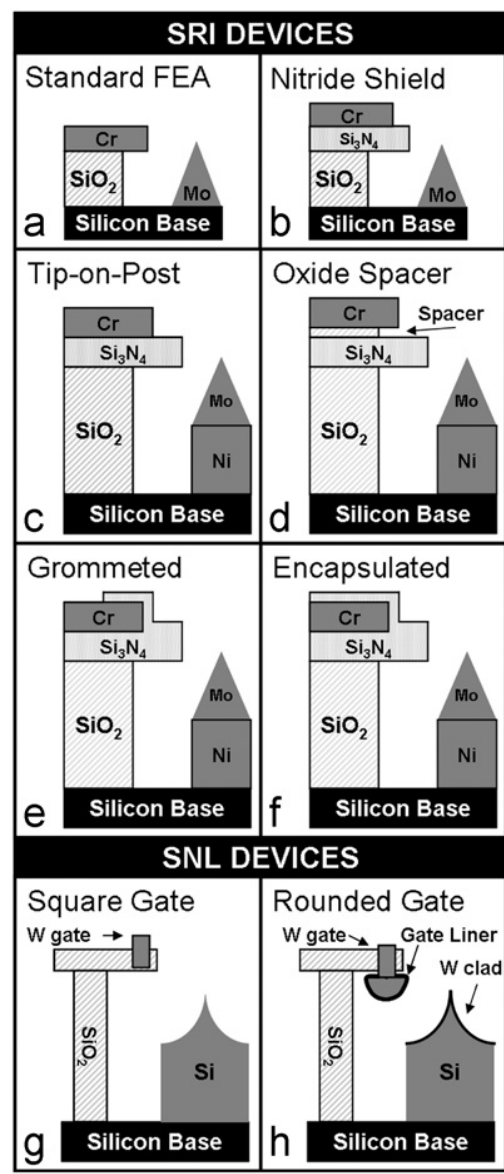

Fig. 3. A cross-sectional view of the array designs tested. (a)-(f) are arrays produced by $S R I$ and $(\mathrm{g})$ and $(\mathrm{h})$ are arrays produced by $S N L$. 
in the gate electrode are centered over the emitter tips. When operating the conventional FEA devices as ion sources, the fields at the gate were sufficient to induce field electron emission which initiated electrical breakdown. The first approach taken to reduce the field at the gate was to introduce a silicon nitride shield, $\mathrm{Si}_{3} \mathrm{~N}_{4}$, under the gate electrode, as in Fig. 3b. The results from initial testing of this device have been previously reported [8]. The $\mathrm{Si}_{3} \mathrm{~N}_{4}$ shield continues to be a design feature, as seen in Fig. 3c-f.

The operating voltages $(>500 \mathrm{~V})$ that were required to achieve desorption fields exceeded that of the breakdown strength of the $\mathrm{SiO}_{2}$ bulk dielectric and tip cavity surfaces of conventional FEA's. To ensure dielectric hold off at $\sim 1000 \mathrm{~V}$, the thickness of the $\mathrm{SiO}_{2}$ was increased from $\sim 0.5 \mu \mathrm{m}$ (Fig. 3a and b) to $\sim 4 \mu \mathrm{m}$ (Fig. 3c-f). As a result of the thicker bulk dielectrics new methods were developed to fabricate the taller tips required to maintain similar gate-to-tip geometry.

The method employed by SRI to produce tall tips was to fabricate a nickel 'post' understructure. Molybdenum tips were then deposited on the nickel posts using similar techniques as those used to create FEA tips [21]. This "tip-on-post" fabrication design is shown in Fig. 3c. Fig. 3d illustrates a similar device, but with a silicon dioxide, $\mathrm{SiO}_{2}$, dielectric spacer added between the gate and nitride shield to suppress high-fields at the shield-gate-vacuum interface.

To further suppress electron emission from the gate, the most recent designs enclose the gate in an $\sim 0.2 \mu \mathrm{m}$ thick layer of dielectric nitride, $\mathrm{Si}_{3} \mathrm{~N}_{4}$, as shown in Fig. $3 e$ and $\mathrm{f}$. In Fig. 3e, a grommet of $\mathrm{Si}_{3} \mathrm{~N}_{4}$ coats the inner edge and top corner of the gate; leaving the top of the gate exposed. In Fig. $3 f$ the entire gate electrode is encapsulated in $\mathrm{Si}_{3} \mathrm{~N}_{4}$.

All SRI arrays were fabricated with molybdenum tips, except the encapsulated devices, Fig. 3f, which were fabricated with either molybdenum tip or tungsten tip varieties.

\subsubsection{Sandia National Laboratories (SNL) fabrication}

SNL arrays have also incorporated both thicker bulk dielectrics and the tall tip design. Fig. 3g illustrates an early SNL design with an integrated (square) tungsten gate and tall silicon tip. The bulk dielectric, $\mathrm{SiO}_{2}$, in the SNL arrays typically ranges from $5 \mu \mathrm{m}$ to $10 \mu \mathrm{m}$ in thickness. The tall tip is produced by etching isolated silicon posts that were defined during the fabrication process [20].

To further reduce the field on the gate, a rounded gate geometry and thin low-stress silicon nitride (LSN) liner was added, as shown in Fig. 3h. Finally, to reduce field penetration into the silicon tips, a tungsten cladding was applied. Details of design, modeling and fabrication of these rounded gate devices have been previously discussed [20]. The most recent SNL fabrication effort has focused on increasing the thickness of the $\mathrm{Si}_{3} \mathrm{~N}_{4}$ gate liner.

\subsubsection{SEM micrographs of SRI and SNL devices}

Fig. 4a and b are SEM micrographs of the most recent devices tested. Fig. 4a is a grommeted gate device developed by SRI based on Fig. 3e. The dielectric material partially encloses the gate structure, and a molybdenum tip has been deposited on a nickel post. Fig. 4b shows a rounded gate device developed by SNL based on Fig. 3h. A $100 \mathrm{~nm}$ thick LSN liner surrounds the gate, and the tip and post are clad with tungsten. SEM imaging of the array structures has determined the radius of the tip apices to be $\sim 20 \mathrm{~nm}$ for both SRI and SNL arrays.

\subsection{Array operating characteristics}

Experimental testing results of the different array designs are shown in Table 1. Here, the operating mode of each design iteration is separated into sub-categories of applied pulsed voltages (for field desorption), and applied d.c. voltages (for field

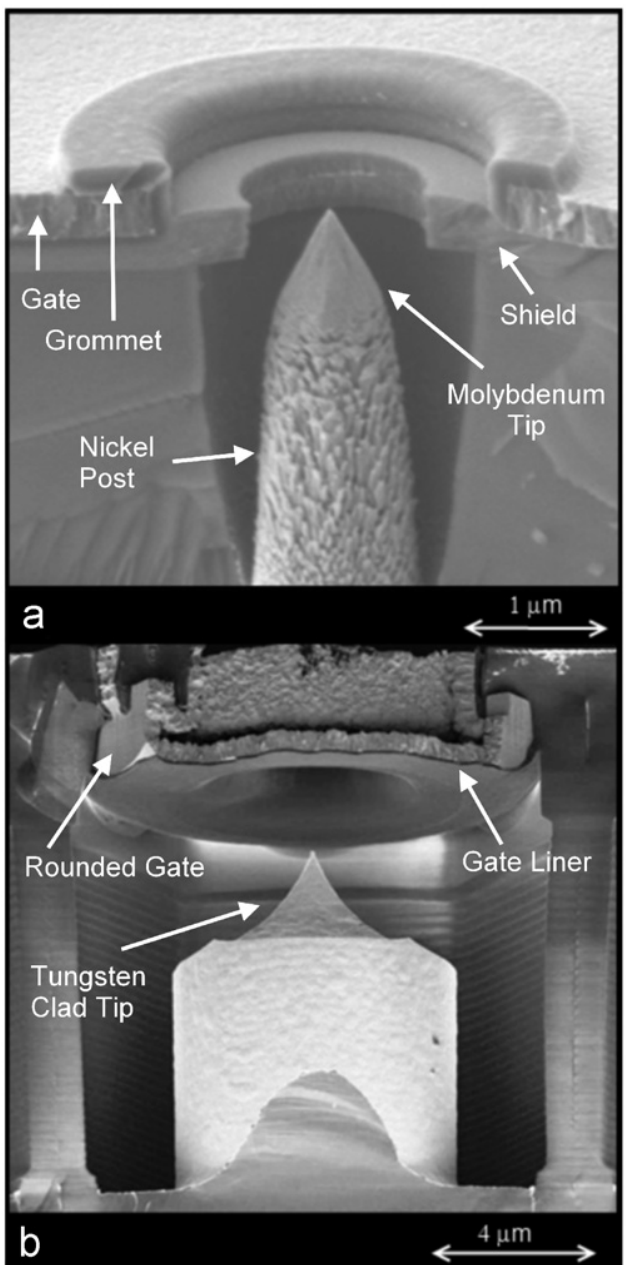

Fig. 4. SEM images of the most recent array design. (a) An SRI grommeted gate array in which a molybdenum tip has been deposited on top of a nickel post. (b) A rounded gate device produced by SNL. The rounded tungsten gate and thin film dielectric gate liner $(100 \mathrm{~nm})$ are indicated with arrows. The tip is tungsten clad silicon.

ionization). For each sub category the mean, standard deviation and maximum applied voltages and inferred fields achieved are reported. Finally, references to the array designs shown in Fig. 3 are included.

The maximum pulsed field (for field desorption) achieved with an SRI array was $36 \mathrm{~V} / \mathrm{nm}$; a maximum of $33 \mathrm{~V} / \mathrm{nm}$ was achieved with an SNL array. The maximum d.c. field achieved (for field ionization) was $24 \mathrm{~V} / \mathrm{nm}$ with an SRI device, and $21 \mathrm{~V} / \mathrm{nm}$ with an SNL device. Both the array types can typically sustain a higher pulsed (20 ns) voltage than a d.c. voltage. There is also a general trend of increasing fields for each of the array iterations tested.

Note that Fig. 3h serves as the array design for most SNL devices. The different SNL iterations listed focus on improving this structure by including a LSN dielectric gate liner. The three rounded gate iterations discussed start with the initial rounded gate (no liner) arrays fabricated, and are then identified by the thickness of the respective gate liner. Finally, early voltage breakdown experiments used a positive $60 \mathrm{~Hz}$ half-wave rectified sinusoidal a.c. applied voltage, as noted by a 'dagger' in the table. There is likely little difference between the a.c. and d.c breakdown voltages (and fields).

\subsection{Single etched-wire tip studies}

Field ionization and field desorption experiments were conducted with single etched-wire tungsten tips of similar radii to 
Table 1

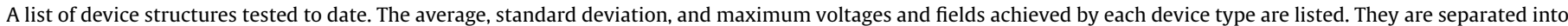
sub-categories of pulsed (field desorption) and d.c. (field ionization) modes.

\begin{tabular}{|c|c|c|c|c|c|c|c|c|c|c|c|c|c|}
\hline \multirow[t]{3}{*}{ Device } & \multicolumn{6}{|c|}{ Pulsed operation } & \multicolumn{6}{|c|}{ D.C. (or A.C.) operation } & \multirow[t]{3}{*}{ Basic structure } \\
\hline & \multicolumn{3}{|c|}{ Voltage (V) } & \multicolumn{3}{|c|}{ Field (V/nm) } & \multicolumn{3}{|c|}{ Voltage (V) } & \multicolumn{3}{|c|}{ Field (V/nm) } & \\
\hline & Avg. & $\pm \sigma$ & Max. & Avg. & $\pm \sigma$ & Max. & Avg. & $\pm \sigma$ & Max. & Avg. & $\pm \sigma$ & Max. & \\
\hline \multicolumn{14}{|l|}{ SRI DEVICES } \\
\hline FEA (Standard) & 204 & 73 & 345 & 7 & 2.2 & 11 & - & - & - & - & - & - & Fig. 3a \\
\hline Nitride shield ${ }^{a}$ & 422 & 93 & 570 & 16 & 4.2 & 27 & - & - & - & - & - & - & Fig. 3b \\
\hline Tip-on-post ${ }^{\mathrm{a}, \mathrm{b}}$ & 525 & 109 & 820 & 18 & 4.4 & 26 & - & - & - & - & - & - & Fig. $3 c$ \\
\hline Dielectric spacer ${ }^{\mathrm{a}, \mathrm{b}, \mathrm{c}}$ & 676 & 144 & 920 & 23 & 5.1 & 32 & $425^{\dagger}$ & 84 & 700 & $13^{\dagger}$ & 2.9 & 21 & Fig. 3d \\
\hline Grommeted gate ${ }^{\mathrm{a}, \mathrm{b}}$ & 1031 & 258 & 1530 & 30 & 4.7 & 36 & - & - & - & - & - & - & Fig. 3e \\
\hline Encaps. gate (Mo tips) $)^{\mathrm{a}, \mathrm{b}}$ & 1013 & 155 & 1250 & 28 & 3.7 & 33 & $827^{*}$ & 175 & 1000 & $22 *$ & 2.4 & 24 & Fig. $3 f$ \\
\hline Encaps. gate (W tips) $)^{\mathrm{a}, \mathrm{b}}$ & $832^{*}$ & 40 & 875 & $29 *$ & 0.9 & 30 & $570^{*}$ & 170 & 690 & $17^{*}$ & 4.2 & 20 & Fig. $3 \mathrm{f}$ \\
\hline \multicolumn{14}{|l|}{ SNL DEVICES } \\
\hline Square gate $\mathrm{e}^{\mathrm{b}}$ & $462 *$ & 237 & 835 & $7 *$ & 3.8 & 14 & - & - & - & - & - & - & Fig. 3g \\
\hline Rounded gate ${ }^{\mathrm{d}}$ & 520 & 205 & 856 & 12 & 4.3 & 18 & - & - & - & - & - & - & Fig. $3 \mathrm{~h}$ \\
\hline $100 \mathrm{~nm}$ gate liner ${ }^{\mathrm{d}, \mathrm{e}, \mathrm{f}}$ & 1018 & 206 & 1175 & 22 & 4.8 & 27 & 730 & 195 & 1350 & 15 & 2.9 & 21 & Fig. $3 \mathrm{~h}$ \\
\hline $150 \mathrm{~nm}$ gate liner $\mathrm{r}^{\mathrm{d}, \mathrm{e}, \mathrm{f}}$ & 842 & 280 & 1200 & 24 & 7.9 & 33 & $560^{*}$ & - & 560 & $13^{*}$ & - & 13 & Fig. $3 \mathrm{~h}$ \\
\hline
\end{tabular}

a Silicon nitride shield.

b Tip-on-post.

c Silicon dioxide spacer.

d Rounded gate.

e Nitride gate liner.

f Tungsten clad tip.

${ }^{\dagger}$ a.c. operation.

* Insufficient data and not statistically significant.

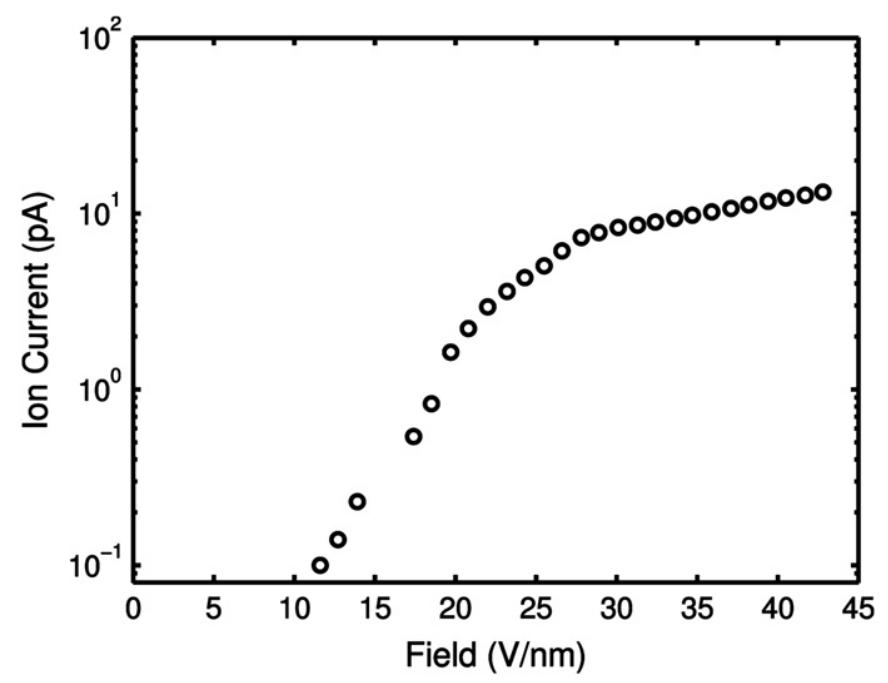

Fig. 5. A semi-log plot showing deuterium field ionization current vs. field from a single etched-wire tungsten tip at $77 \mathrm{~K}$.

those of the arrays. These studies were used to quantify potential array deuterium ion yields on a per-tip basis.

\subsubsection{Field ionization studies}

For field ionization experiments, the radius of the etched-wire tip was, $r_{\text {tip }}=16.8 \mathrm{~nm}$, and was operated in identical conditions as those used for field ionization studies of arrays.

Fig. 5 is a semi-log plot of deuterium ion current vs. tip field from the single etched-wire tungsten tip at $77 \mathrm{~K}$ in a deuterium atmosphere of $3.7 \times 10^{-4}$ Torr. The shape of the curve is in good agreement with studies conducted by Van Eekelen [22] where the 'knee' in the plot of Fig. 5 represents a transition from ionization rate limited current to gas supply limited current. The location of the 'knee' depends on operational pressure and temperature. A further increase in field beyond that in the plot leads to evaporation of tip material at $\sim 45 \mathrm{~V} / \mathrm{nm}$ for tungsten when promoted by the presence of hydrogen [9].

At fields of $20 \mathrm{~V} / \mathrm{nm}$, the beam current is $\sim 99 \%$ molecular deuterium ions, $\mathrm{D}_{2}^{+}$. [23,24]. As the field is increased, dissociative ionization of molecular deuterium ions occurs, or $\mathrm{D}_{2}^{+} \rightarrow \mathrm{D}^{+}+\mathrm{D}$, of which the remaining $D$ atom can again field ionize [23]. For fields above $40 \mathrm{~V} / \mathrm{nm}$, we expect primarily atomic deuterium ion production, with a lower bound of $\sim 75 \% \mathrm{D}^{+}-$to- $\mathrm{D}_{2}^{+}$at a field of $40 \mathrm{~V} / \mathrm{nm}[23,24]$.

\subsubsection{Field desorption studies}

Deuterium desorption was investigated to determine the maximum deuterium ion yield possible from a single tip. Measurement of the number of ions contained in a desorption pulse is not straightforward, and thus the number of deuterium ions per desorption pulse were quantified using titanium film evaporation. In this case, known thicknesses of titanium film were evaporated from the surface of an etched-wire tungsten tip, such that the titanium peak height in the TOF spectra corresponded to the amount of titanium contained in the film. The absolute number of $\mathrm{D}^{+}$ions desorbed from a single tip is then inferred by comparing the deuterium peak height to that of the titanium peak height in the TOF spectra. Note that titanium film evaporation in this manner is dominated by $\mathrm{Ti}^{2++}$ ions and that contributions from $\mathrm{Ti}^{+}$and $\mathrm{Ti}^{3+}$ charged ions is negligible [15]. This approach to absolute charge calibration using titanium films has been discussed previously [15].

Fig. 6 shows transposed TOF spectra for three different experiments conducted with a tip of radius $18.6 \mathrm{~nm}$ at $77 \mathrm{~K}$. The TOF data have been time-shifted for peak height comparison purposes, and no longer represents arrival times; however, the relative peak widths in flight time are maintained. The first two experiments, represented by the dashed and dotted lines, show the resulting titanium peak heights corresponding to the evaporation of 2.5 atomic layers and 4.5 atomic layers of titanium film, respectively. The second $y$-axis represents the total number of $\mathrm{Ti}^{2+}$ ions evaporated assuming a $45^{\circ}$ half-angle of emission from the single etched-wire tip. The third experiment represents 


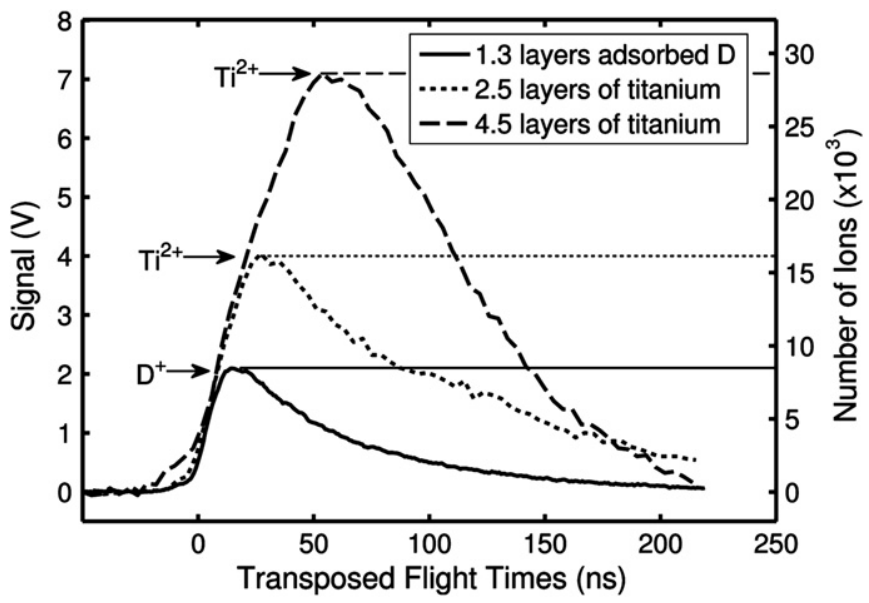

Fig. 6. Overlain TOF peaks produced by evaporating titanium films and desorbing deuterium from a single etched-wire tungsten tip. The solid line represents the desorption of $\sim 1.3$ layers of deuterium from the etched wire tip. The right $y$-axis shows the correspondence between the peak height and the number of ions produced.

deuterium desorption from the etched-wire tip (no titanium deposited), and shows the resulting $\mathrm{D}^{+}$peak height. The $\mathrm{D}^{+}$peak height, when compared against the $\mathrm{Ti}^{2+}$ peak heights, indicates that $\sim 8400$ deuterium ions are produced by single etched-wire tip. This value corresponds to desorption of $\sim 1.3$ atomic layers of deuterium.

\subsection{Array ion current and neutron production using field ionization}

The arrays tested for field ionization studies were 10,267 tip, $1.5 \mathrm{~mm}^{2}$ SNL rounded gate arrays with $100 \mathrm{~nm}$ LSN gate liners, as shown in Fig. 4b. The ionization experiments were conducted at $77 \mathrm{~K}$ in a deuterium atmosphere of $3.7^{2+} \times 10^{-4}$ Torr. Fig. $7 \mathrm{a}$ is an optical micrograph of an SNL rounded gate array and shows the hexagonally packed tip arrangement of the array emission area. These studies correspond to the d.c. fields shown in Table 1 for the $100 \mathrm{~nm}$ gate liner devices.

\subsubsection{Field ionization and field electron emission patterns}

Experiments were conducted to investigate both ion emission half-angle and ion beam uniformity. The half-angle of emission was observed and calculated by positioning an array close enough to the detector to ensure that the entire emission cone was captured. Photographs of the CEMA outputs for electron and ion emission patterns are shown in Fig. $7 \mathrm{~b}$ and $\mathrm{c}$, respectively. Note the hexagonal shape and orientation of the emission surface, shown in Fig. 7a, is visible in Fig. 7b and, although less apparent, in Fig. 7c. The image of the deuterium ion emission shown in Fig. 7c was created with $\sim 1 \mathrm{nA}$ of field ion current; the inner circular area defined by $r_{3}$ was due to saturation of the CEMA.

The majority of ion and electron emission is contained within the circular area defined by the radius, $r_{1}$, and corresponds to an array emission half-angle of $\sim 34.5^{\circ}$. By examining SEM micrographs of the SNL array devices we determined the gate hole transmission angle to be $\sim 33^{\circ}$, which is in good agreement with the measured half-angle.

For this type of array, the gate transmission hole is the limiting factor for the array emission half-angle and there is likely some interception of ions by the gate electrode. It is unknown what effects this interception has on array operation. However, modeling shows that when the array tips are in the presence of a target field and properly positioned relative to the gate electrode plane,
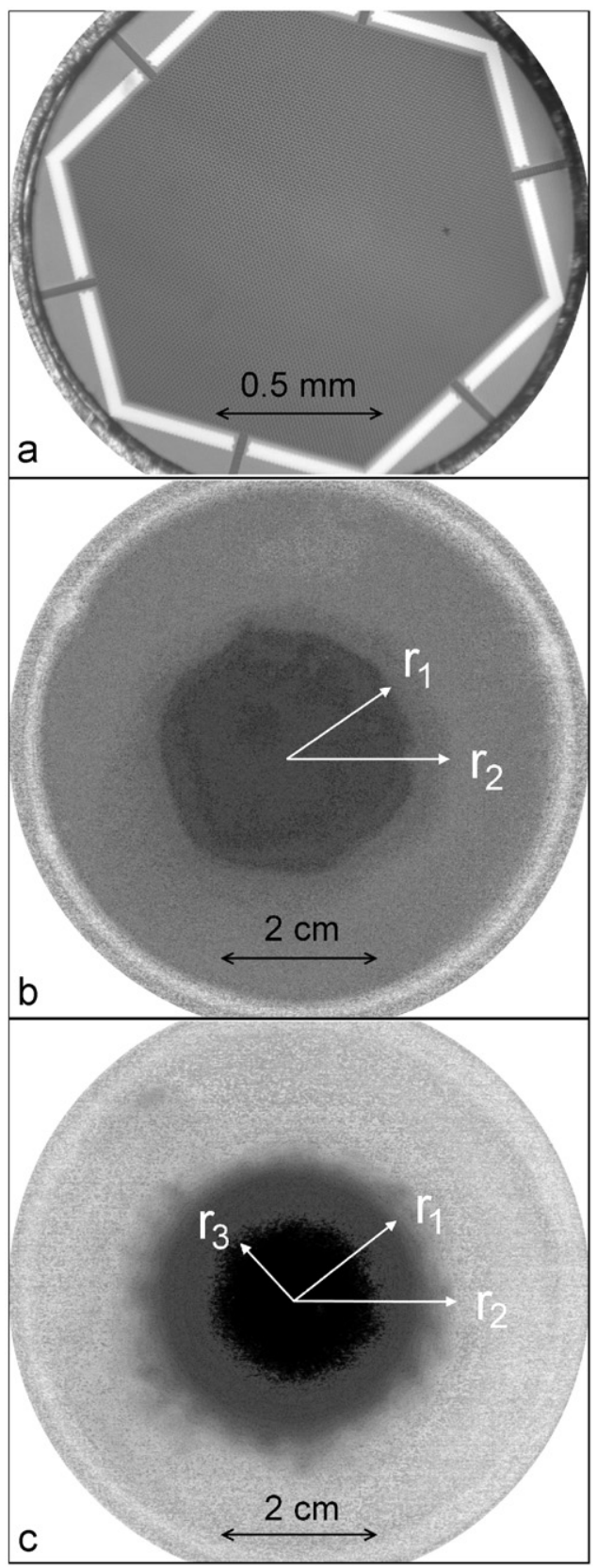

Fig. 7. Correlation of the array emission area shape with the electron and ion emission patterns. (a) An optical micrograph of the emission area of an SNL rounded gate device (see Fig. 4b). (b) and (c) show, respectively, photographs of the electron and field ionization emission patterns from an array. In (b) and (c) the majority of emission appears within a radius $r_{1}$ of the array center and does not extend beyond radius $r_{2}$. In (c), $r_{3}$ is due to saturation of the phosphor screen. The spatial orientation of the array is the same in (a)-(c). The hexagonal tip packing pattern of the array in (a) can be seen in (b), and is present, but less apparent in $(c)$.

the target field prevents interception of ions by the gate. This will be presented in a future publication on modeling results [19].

Note that the image in Fig. $7 \mathrm{c}$ is azimuthally uniform, and suggests that the arrays are emitting evenly. Also, Fig. 7b (not apparent, but visible during the experiment) and 7c show there is a radial gradient in both electron and ion emission currents. In Fig. 7c, the ion current is most intense at the center, and decreases toward the edge of the circle defined by $r_{1}$. Why this is remains unknown. Future studies will investigate this effect, and attempt to explain why it is happening. Beyond $r_{1}$, edge effects appear as spotty regions, and the current drops quickly to zero by $r_{2}$. 

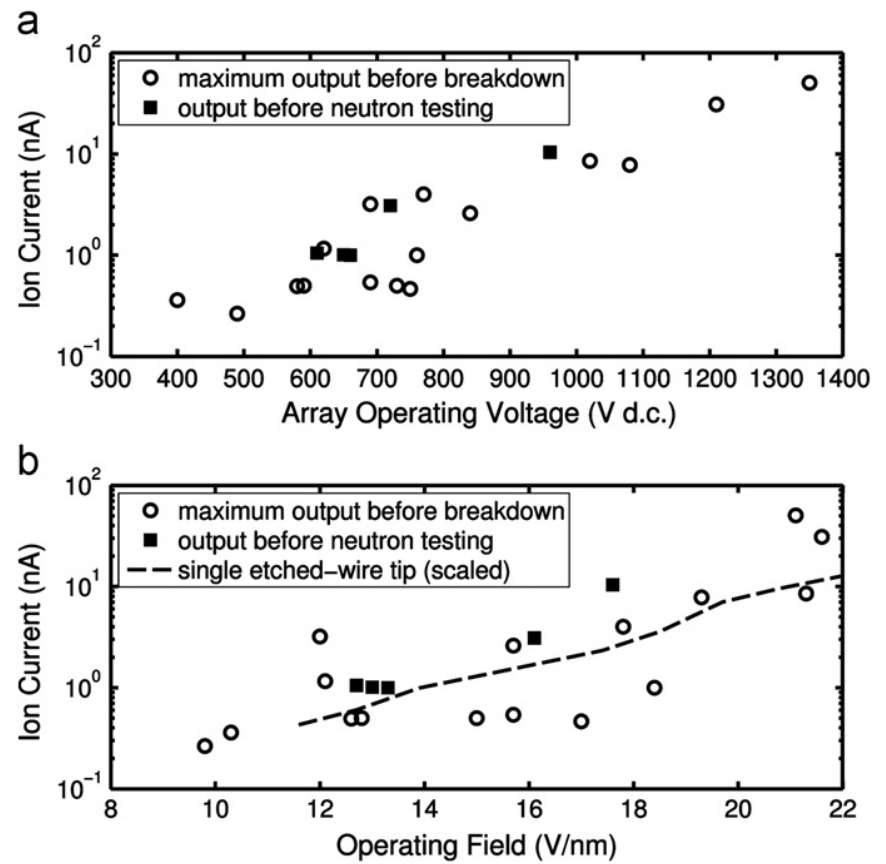

Fig. 8. Maximum field ion current vs. (a) array operating voltage and (b) operating field for different SNL rounded gate arrays. The single etched-wire tip data (from Fig. 5), is scaled and plotted for comparison.

\subsubsection{Deuterium field ionization currents}

The plot in Fig. 8a is a semi-log plot of the maximum ion current yield vs. array operating voltage. The circular data points show the maximum ion current measured; with the best array outputting $\sim 50 \mathrm{nA}$ of deuterium ion current. The filled squares show the maximum ion current measured before moving the array to the light ion accelerator system for neutron production experiments. Fig. 8b shows the ion current yield (Fig. 8a) vs. calculated field. Note that the array data are consistent with onset fields for deuterium field ionization $(\sim 10 \mathrm{~V} / \mathrm{nm})$. Also recall that typically individual arrays have different voltage-field calibration factors which causes the data to shift in Fig. 8b.

Also plotted in Fig. 8b, represented by the dashed line, are the single etched-wire tip data from Fig. 5. The ion current data from Fig. 5 have been scaled by the number of tips $(10,267)$ and then reduced by a factor of 0.42 to account for the $33^{\circ}$ emission halfangle of the arrays, relative to the $45^{\circ}$ half-angle of the etchedwire tip. We see that the array field ionization data agree quite well with that of the scaled single etched-wire tip data, indicating that each of the array tips are contributing equally on a per-tip basis, and thus, array fabrication is reasonable uniform between array tips.

\subsubsection{Neutron production}

Neutron output rates were measured for SNL arrays operating in the field ionization mode. Fig. 9 shows the performance from a single SNL array of both the ion current vs. operating voltage and field, and the measured neutron yield. The maximum production of $10 \mathrm{nA}$ at $960 \mathrm{~V}$ corresponds to a field of $17.7 \mathrm{~V} / \mathrm{nm}$. The neutron yield expected for the given ion current, assuming $2.65 \times 10^{4} \mathrm{n} / \mu \mathrm{C}$ of $\mathrm{D}_{2}^{+}$at $90 \mathrm{kV}$ (45 kV/nucleon) using a $\mathrm{TiD}_{2}$ target [25], is shown on the second $y$-axis. The actual measured neutron yield versus array operating voltage (and field) is shown as open squares, and indicates neutron production is consistent with a beam of molecular deuterium ions. The maximum measured neutron yield

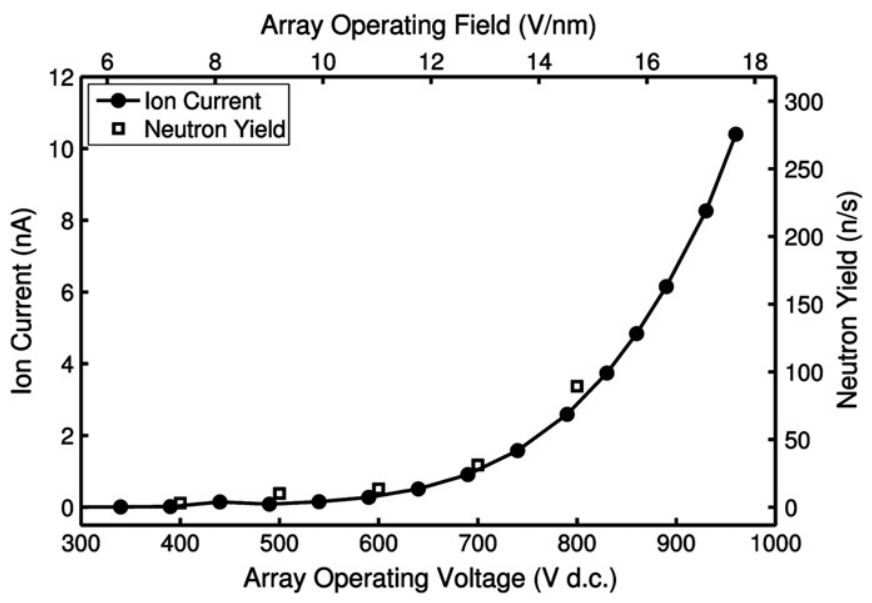

Fig. 9. Measured field ion current from an SNL rounded gate array vs. array operating voltage and field. The right $y$-axis corresponds to calculated neutron production for both a given ion current (solid line with circles) and the measured neutron yield (squares).

for this array before breakdown was $89 \mathrm{n} / \mathrm{s}$, operating at $800 \mathrm{~V}$ and at a field of $14.7 \mathrm{~V} / \mathrm{nm}$. The highest measured neutron production observed from this type of array was $95 \mathrm{n} / \mathrm{s}$ at a field of $17 \mathrm{~V} / \mathrm{nm}$.

\subsubsection{Theoretical neutron yield}

From the single etched-wire tip results in Sec. 3.3.1, for a field of $40 \mathrm{~V} / \mathrm{nm}$, we expect an ion current of $\sim 10 \mathrm{pA} /$ tip, and a beam composed primarily of $\mathrm{D}^{+}$. At present, arrays contain $\sim 10^{6}$ tips/ $\mathrm{cm}^{2}$, and increased tip packing densities of $10^{7} \mathrm{tips} / \mathrm{cm}^{2}$ should be realizable. If each of the array tips operates as a single tip, we would expect roughly $100 \mu \mathrm{A} / \mathrm{cm}^{2}$ of $\mathrm{D}^{+}$ion current from an array. Assuming a D-D neutron yield of $\sim 4 \times 10^{5} \mathrm{n} / \mu \mathrm{C}$ (at $120 \mathrm{kV}$ ) for $\mathrm{D}^{+}$[25] we expect a neutron output of $\sim 4 \times 10^{7} \mathrm{n} / \mathrm{s} / \mathrm{cm}^{2}$. For a D-T neutron yield of $\sim 1 \times 10^{8} \mathrm{n} / \mu \mathrm{C}$ (at $120 \mathrm{kV}$ ) [25], an output of $\sim 1 \times 10^{10} \mathrm{n} / \mathrm{s} / \mathrm{cm}^{2}$ is achievable. This could be increased further by optimizing deuterium gas pressures, operating at temperatures lower than $77 \mathrm{~K}$ and increasing the array area.

\subsection{Array field desorption mass spectra}

The arrays tested for these field desorption studies were SNL rounded gate devices with $100 \mathrm{~nm}$ and $150 \mathrm{~nm}$ gate liners (Fig. 3h), SRI grommeted gate devices (Fig. 3e), and SRI encapsulated gate devices (Fig. 3f). Arrays were tested at $77 \mathrm{~K}$ in a deuterium atmosphere of $3.7 \times 10^{-5}$ Torr which allowed for ample deuterium re-adsorption between the applied desorption pulses at $1 \mathrm{~Hz}$. The signal intensities of the TOF data are generally not relative to one another because of different gain settings on the CEMA, and the varying number of tips in the different array types. Surface contaminants such as $\mathrm{C}^{2+}, \mathrm{C}^{+}, \mathrm{O}^{+}$and $\mathrm{CO}^{+}$that appear in the TOF spectra are due to environmental sources that arise from fabrication and handling the arrays prior to testing.

\subsubsection{Onset of deuterium desorption and contribution from field ionization}

The rounded gate devices produced by SNL (Fig. 3f), and the dielectric grommeted (Fig. 3g) and encapsulated devices (Fig. 3h) from SRI, have consistently achieved fields beyond those required for the onset of deuterium desorption ( $>20 \mathrm{~V} / \mathrm{nm}$ ). Fig. 10 shows two TOF mass spectra from an SNL rounded gate array $(10,267$ tips with a $100 \mathrm{~nm}$ gate liner) at the onset of deuterium desorption. The solid line is a spectrum at a field near the 


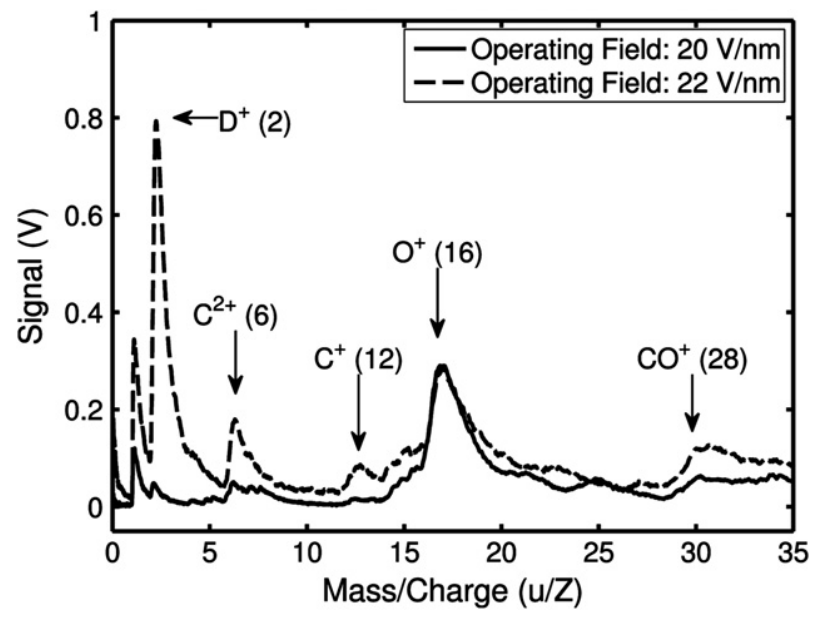

Fig. 10. Mass spectra from an SNL rounded gate array showing the onset of deuterium desorption and the increase in deuterium peak height with increasing electric field.

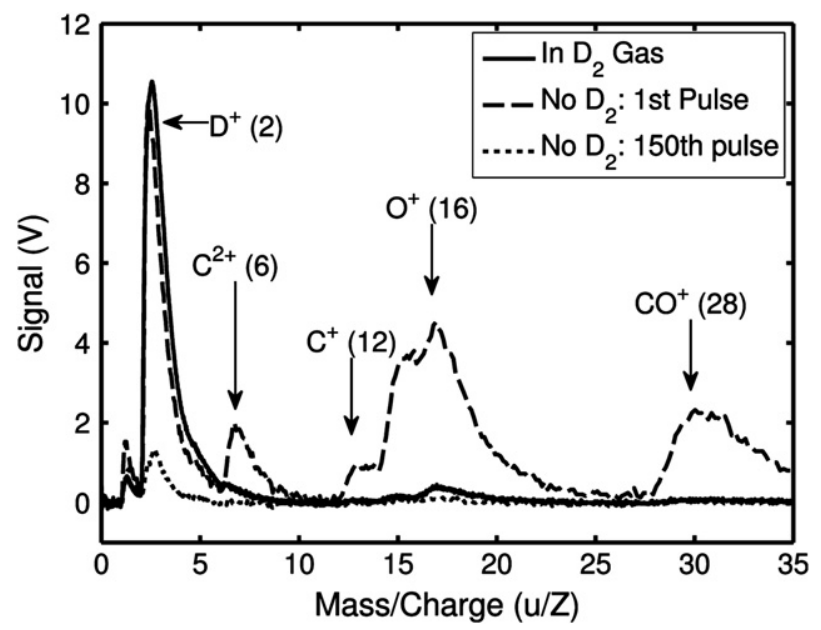

Fig. 11. Mass spectra from an SNL rounded gate array illustrating that the major contribution to the deuterium peak are ions generated by field desorption (and not field ionization). The first pulse after deuterium gas is removed shows a large desorption signal. Since there is no longer gas in the system, the surface is not re-supplied with deuterium, and the $\mathrm{D}^{+}$peak has decreased significantly by the 150th pulse.

deuterium desorption threshold and consists of typical surface contaminants. When the field is increased to $22 \mathrm{~V} / \mathrm{nm}$ the $\mathrm{D}^{+}$ signal becomes the predominant peak in the spectrum, as shown by the dashed line in Fig. 10.

Since the desorption fields for deuterium exceed those necessary for field ionization, there may be a contribution to the $\mathrm{D}^{+}$peak due to field ionization during the 20 ns desorption pulse. This contribution was determined by comparing deuterium peak heights from an array when pulsed in a deuterium atmosphere, to that of the array pulsed after removal of the gas from the system, as shown in Fig. 11. The solid line is the signal produced from a 10,267-tip array when pulsed in a deuterium atmosphere. The dashed line is the first pulse after deuterium adsorption on the tip and removal of the gas, and resulted in a $3.7 \%$ decrease in the deuterium peak height. This demonstrates that only a small contribution of the deuterium signal is from field ionization. By the 150th pulse, the deuterium peak has decreased to $13 \%$ of its original value. Refilling the test system with deuterium returns the signal to that of the solid line. For each case, the pulsed field was $27 \mathrm{~V} / \mathrm{nm}$.

Note in Fig. 11, the dramatic increase in surface contaminant peak heights in the TOF spectrum after removing gas from the system. It is assumed that these contaminants are the result of surface diffusion from the tip shanks to the tip apices during the time taken to vent gas from the system ( $\sim 2 \mathrm{~min})$. Although operation at liquid nitrogen temperature should suppress thermal diffusion to a large extent, it is likely that diffusion is still field driven, as a result of the d.c. holding voltage on the array tips (which was maintained during evacuation). The contaminants are still present in the other spectra, however, the peak heights are significantly less, as the pulse frequency $(1 \mathrm{~Hz})$ limits the time contaminants have to diffuse before they are removed. This field driven diffusion is also the likely reason why the deuterium peak has not completely disappeared by the 150th pulse, even though there is no means of resupply from the atmosphere.

\subsubsection{Deuterium, metal and metal oxide desorption from arrays}

The maximum fields for deuterium desorption from molybdenum tips, without removing tip substrate material are $\sim 35 \mathrm{~V} / \mathrm{nm}$ [9]. Fig. 12 shows the normalized TOF mass spectra from a single etched-wire molybdenum tip and an SRI grommeted gate 109 tiparray, pulsed at fields high enough to observe molybdenum tip substrate evaporation. The dashed line shows deuterium desorption and the relative abundance of $\mathrm{Mo}^{3+}$ (32) and $\mathrm{Mo}^{2+}$ (48), the dominant charge species formed by evaporation of the molybdenum tip substrate [9], removed from a single etchedwire tip pulsed at a field of $38 \mathrm{~V} / \mathrm{nm}$. The solid line shows the mass spectrum from an array pulsed at $36 \mathrm{~V} / \mathrm{nm}$ and is in good agreement with the spectra of the single etched-wire tip. This is the highest tip field achieved from an array to date.

Molybdenum oxides and surface contaminants $\left(\mathrm{C}^{2+}, \mathrm{C}^{+}, \mathrm{O}^{+}\right.$ and $\mathrm{CO}^{+}$) are present in the array spectrum, but not in the single etched-wire tip spectrum because the etched-wire tip was flash heated (in situ, to $\sim 2000{ }^{\circ} \mathrm{C}$ ) and then field evaporated to provide an atomically clean molybdenum surface. We are unable, as of yet, to clean the array tip surfaces in this manner.

Fig. 13 shows the desorption spectrum from the same SRI grommeted gate (109-tips) array in Fig. 12 compared to that of an SRI encapsulated gate $(15,405$ molybdenum tips) array, pulsed at lower fields. Note that surface contaminant and oxide mass peaks are consistent between the two types of molybdenum tip arrays. Also, at the lower fields, the relative intensities of the oxides are more prevalent suggesting that a large quantity of oxides were already removed by the time the higher operating fields were achieved with the grommeted gate array.

The desorption of tungsten oxide tip material has also been observed from tungsten tipped arrays. Fig. 14 shows TOF mass

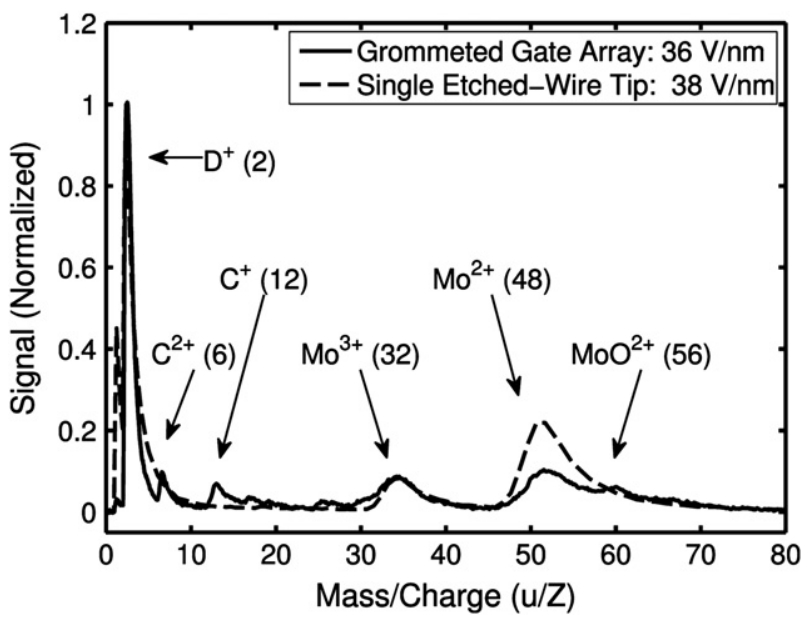

Fig. 12. Mass spectra comparing molybdenum metal evaporation from a single etched-wire tip and an SRI grommeted gate array. 


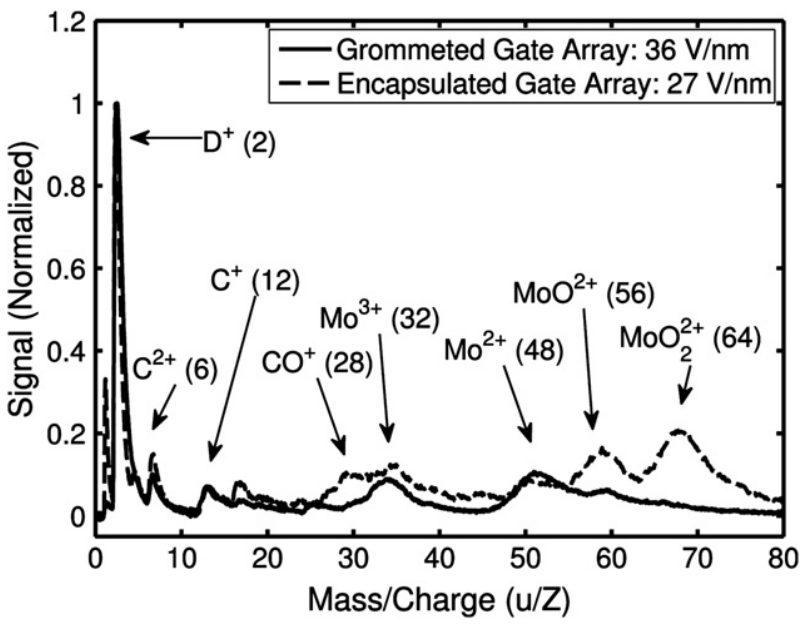

Fig. 13. Mass spectra showing the desorption of molybdenum and molybdenum oxide from an SRI grommeted gate array and an SRI encapsulated gate array.

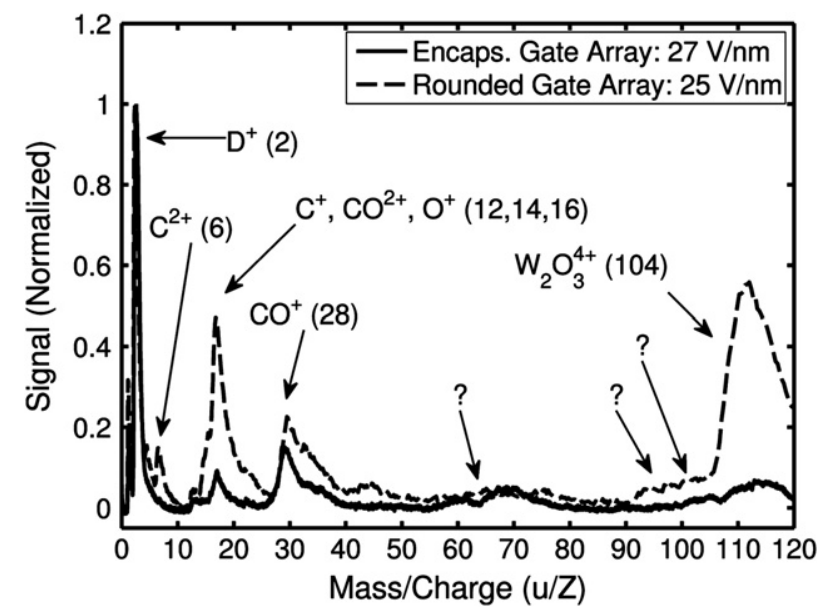

Fig. 14. Mass spectra of tungsten oxide desorption from an SNL rounded gate array and an SRI encapsulated gate array. Tungsten clad array tips and deposited tungsten array tips produce similar mass peaks.

spectra from an SNL rounded gate array (150 nm gate liner, 10,267-tips) with tungsten clad tips and an SRI encapsulated gate (25,693- tips) array with deposited tungsten tips. The tungsten oxide peak, $\mathrm{W}_{2} \mathrm{O}_{3}^{4}$, at mass-to-charge 104 , is present in both spectra. Again, typical surface contaminants, such as $\mathrm{C}^{2+}, \mathrm{C}^{+}, \mathrm{O}^{+}$ and $\mathrm{CO}^{+}$, appear. Other unknown mass peaks also appear between mass-to-charge ratios of 60 to 70 , and 90 to 110 . These may be the result of tungsten carbides and oxides, including: $\mathrm{WC}^{3+}$ (65), $\mathrm{WO}^{3+}(67), \mathrm{WCO}^{3+}(70), \mathrm{W}^{2+}(92), \mathrm{WC}^{2+}(98)$, $\mathrm{WO}^{2+}(100)[26]$.

\subsubsection{Number of deuterium ions desorbed from arrays}

Using the single etched-wire tip experiments discussed in Sec. 3.3.2, we can quantify the number of deuterium ions desorbed from an array. Fig. 15 shows the $\mathrm{D}^{+}$peak signal height of a 10,267 tip SNL rounded gate array (150 $\mathrm{nm}$ gate liner) compared to that of the single etched-wire tip. The wider $\mathrm{D}^{+}$peak width for the array is a result of the lower operating voltage, and thus longer ion arrival time. The second $y$-axis is calibrated assuming an emission halfangle of 45 degrees. The deuterium peak height from the SNL rounded gate array corresponds to 11,000 ions hitting the detector per desorption pulse, as compared to the 8400 ions produced by a single tip. These results indicate that the absolute ion desorption

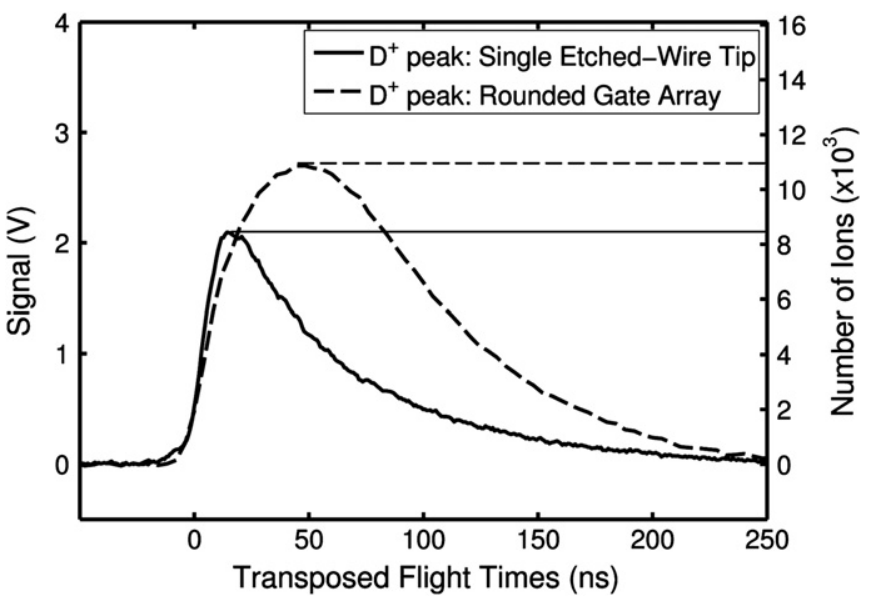

Fig. 15. Deuterium ion desorption of an SNL rounded gate array compared to that of a single etched-wire tip. Low desorption ion yields are the result of array tip surface contamination limiting deuterium adsorption.

from an array is low, and that the $\sim 10,000$ tip array produced only $30 \%$ more ions total than that of a single etched-wire tip.

Low deuterium ion yield from the array is likely the result of surface contamination, which limits the deuterium coverage. The presence of surface contamination is evident in spectra of Figs. 10-14. As mentioned before, etched-wire tips benefit from heating and field evaporation cleaning procedures that yield an atomically clean surface onto which deuterium can readily adsorb. High temperature heating $\left(>900^{\circ} \mathrm{C}\right.$ ) can destroy the arrays due to differing coefficients of thermal expansion for the various materials. To date, we have been able to heat the array up to $\sim 600{ }^{\circ} \mathrm{C}$, and have observed reduced contamination and increased $\mathrm{D}^{+}$signals. Also, we have not yet reached fields that would allow cleaning by field evaporation of array tip substrate material ( $\sim 44 \mathrm{~V} / \mathrm{nm}$ for Mo, $\sim 55 \mathrm{~V} / \mathrm{nm}$ for $\mathrm{W})$. Currently, we are investigating other methods of cleaning array tip surfaces, such as in situ hydrogen plasma treatment.

\subsubsection{Theoretical neutron yield}

Proper implementation of cleaning methods should increase the deuterium yields from arrays to a level which scales per-tip with that presently observed using single etched-wire tips. Here, the maximum neutron production from an array is calculated based on the single etched-wire tip desorption results (at $77 \mathrm{~K}$ ) in Section 3.3.2. From a single etched-wire tip we expect a total $\mathrm{D}^{+}$ion production of $\sim 10^{4}$ ions/pulse/tip for a tip radius of $\sim 20 \mathrm{~nm}$. Increased tip packing densities of $10^{7}$ tips $/ \mathrm{cm}^{2}$ should be realizable. Assuming each of the array tips operate as a single tip, we expect $\sim 10^{11}$ ions/pulse $/ \mathrm{cm}^{2}$, or $\sim 10^{-2} \mu \mathrm{C} /$ pulse $/ \mathrm{cm}^{2}$ from these arrays. At $10^{-2}$ Torr, a pulsing rate of $1 \mathrm{kHz}$ allows for deuterium re-adsorption between pulses (the sticking probability of deuterium on tungsten is 0.1 ) At $1 \mathrm{kHz}$, an array will output $\sim 10 \mu \mathrm{C} / \mathrm{s} / \mathrm{cm}^{2}$ of $\mathrm{D}^{+}$ion current. Assuming a D-D neutron yield of $4 \times 10^{5} \mathrm{n} / \mu \mathrm{C}$ (at $120 \mathrm{kV}$ ) for $\mathrm{D}^{+}$ [25], we expect a neutron output of $\sim 4 \times 10^{6} \mathrm{n} / \mathrm{s} / \mathrm{cm}^{2}$. For a D-T production rate of $\sim 10^{8} \mathrm{n} / \mu \mathrm{C}$ (at $120 \mathrm{kV}$ ) [25], the neutron yield becomes $\sim 1 \times 10^{9} \mathrm{n} / \mathrm{s} / \mathrm{cm}^{2}$. This could be increased further by tiling the array structures. Also because the surface area of a tip scales as $r^{2}$ and the operating voltage scales as $r$, a slight increase in tip radius can significantly increase desorption yields with only a small increase in operating voltage.

\subsection{Electrical breakdown of arrays}

Field electron emission from the gate electrodes is the major source of emitter failure as it initiates electrical breakdown in the 


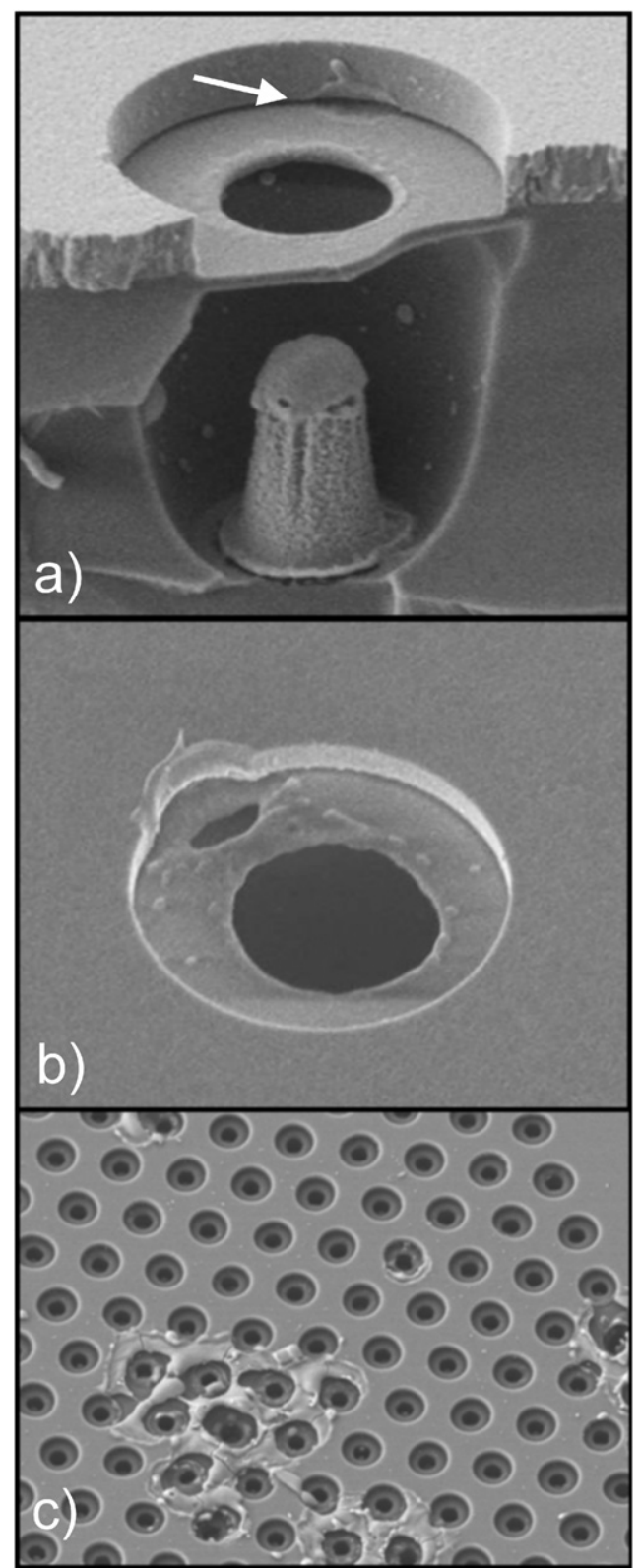

Fig. 16. SEM images of damage to array structures caused by the gate field electron emission. (a) The likely origin of electron emission from the gate is indicated by the arrow, and can lead to tip damage. (b) A hole in the dielectric shielding due to electron emission. (c) Catastrophic damage to a large area of the array.

array structures and can render the array inoperable due to an electrical short. Modeling of array structures has shown that even with idealized, atomically smooth electrode surfaces, electron emission fields exist on parts of the gate electrode once tip fields approach 20-30 V/nm [19].

Currently, arrays are tested until electrical breakdown occurs and the device become inoperable. Varying degrees of damage to the array emitting area is observed, and can range from a small region of damage consisting of only a few tips, to damage across the entire emitter surface. Below, we provide some examples of damage caused during breakdown that is consistent with electron emission from the gate electrode, as observed in post-mortem SEM examination.

Fig. 16a and b are SEM micrographs of individual array tips in a dielectric shielded device (Fig. 3b) exhibiting damage after breakdown. Fig. 16a shows a melted tip after electrical breakdown. Damage at the gate-shield-vacuum interface (triple point) has been

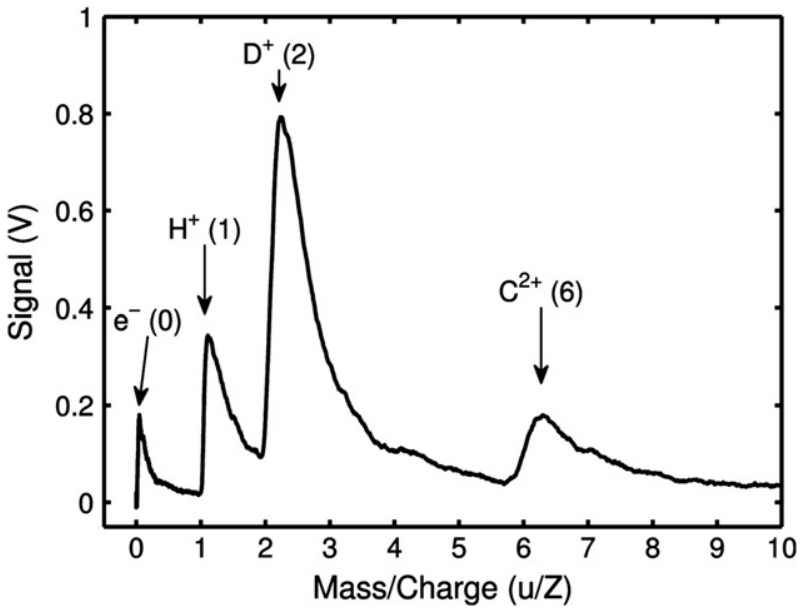

Fig. 17. An electron peak appearing in a TOF mass spectrum while pulsing an SNL rounded gate array at a field of $22 \mathrm{~V} / \mathrm{nm}$.

indicated with an arrow, and is the suspected source of the gate emitted electrons. These electrons can avalanche across the surface of the dielectric shield, driven toward the tip by the applied field, and damage the tip by electron bombardment. Fig. 16b shows another image of damage attributed to gate electron emission. The deformed area of the gate is the likely source of the electrons, and in this instance, the electrons punched a hole directly through the dielectric shield. Fig. 16a and b illustrate the effects of the gate emitted electrons in localized regions, which can trigger more catastrophic breakdowns, as shown in Fig. 16c.

At the current state of fabrication, it is possible for damage to a single tip to electrically short the entire array. Ultimately, incorporating a fusing scheme into fabrication of the emitter array will prevent failure of the entire array when one or a group of tips fail.

More evidence of field electron emission from the gate has also been observed in the TOF mass spectra. In this case, as some threshold voltage was applied to a given array, an electron peak would appear in the spectrum at mass-to-charge 'zero', as in Fig. 17. This electron peak would then typically increase in height with the applied voltage. These electrons are likely secondary electrons produced by gate emitted electrons striking, for example, the dielectric shield in Fig. 16. The appearance of the electron peak was very often a precursor to electrical breakdown of the array.

\section{Conclusions and future work}

The ion source arrays differ greatly from that of the FEA on which they are based as a result of the thicker bulk dielectrics, improved dielectric shielding and the integration of taller tips. The ion source array design evolution was driven by the need to achieve higher tip operating fields and increase the tip-to-gate field ratio in order to suppress field electron emission from the gate. This electron emission ultimately limits the tip operating field by initiating electrical breakdown in the array.

Ongoing fabrication activities have focused on further reducing gate fields by rounding the gate electrode edge and covering it with dielectric material. Successful modifications will allow for increased operating fields and should soon achieve levels optimal for deuterium desorption.

Experiments conducted at $77 \mathrm{~K}$ with applied d.c. voltages have demonstrated field ionization from fabricated arrays. SNL array structures of 10,267 tips in $1.5 \mathrm{~mm}^{2}$ have produced deuterium ion currents upwards of $50 \mathrm{nA}$. This is in good agreement with single etched-wire tip studies. Neutron production studies using field 
ionization from the same arrays have demonstrated neutron yields of $\sim 10^{2} \mathrm{n} / \mathrm{s}$ with the $\mathrm{D}-\mathrm{D}$ fusion reaction at $90 \mathrm{kV}$ in $3.7 \times 10^{-4}$ Torr of deuterium gas.

Field desorption studies conducted at $77 \mathrm{~K}$ have demonstrated the pulsed desorption of deuterium, with onset fields of $\sim 20 \mathrm{~V} / \mathrm{nm}$ consistently being achieved by arrays. The primary output of $\mathrm{D}^{+}$, as seen in the TOF spectra in Figs. 10-14, is often accompanied by desorption of other mass species, typically involving compounds of carbon, oxygen, and oxides of the tip substrate.

In the case of field desorption, contaminants have a significant impact on the deuterium ion output of arrays, as they inhibit deuterium adsorption from gas-phase.

Effective cleaning of the tip surfaces will have a large impact on ion, and thus neutron, yield. Hydrogen plasma treatment of the tip surfaces is being investigated, and field evaporation of the tip surfaces (desorption of the metal tip substrate) will be studied once the fields required are achieved. In contrast, field ionization is largely insensitive to surface contamination as deuterium is ionized in gas phase from a volume in front of the tip, as opposed to being desorbed from the tip surface.

Calculations indicate that the arrays have the potential to provide high neutron outputs in both the pulsed desorption and d.c. field ionization modes at $77 \mathrm{~K}$. For both cases, this type of ion source offers better efficiency and lower power consumption than existing compact neutron generators, which is desirable for the application to fieldable (man-portable) interrogation systems. Future studies will involve characterizing field desorption and field ionization from arrays at room temperature, as well as demonstrating neutron production with field desorption.

\section{Acknowledgments}

This project is a collaboration between The University of New Mexico, SRI International, Sandia National Laboratories and Idaho National Laboratory and is supported by the US Department of Energy through the National Nuclear Security Administration's Office of Nonproliferation and Verification Research and Development (NA-22). The authors wish to acknowledge both Birk Reichenbach and Sid Solano for preliminary experimental work conducted.

\section{References}

[1] Report of the Defense Science Board Task Force, Office of the Undersecretary of Defense for Acquisition, Technology and Logistics, Preventing and
Defending Against Clandestine Nuclear Attack, U.S. Department of Defense, Washington, DC, June 2004.

[2] For example, D. Slaughter, et al., Lawrence Livermore National Laboratory Report UCRL-ID-155315, 2003.

[3] Committee on Science and Technology for Countering Terrorism, Nationa Research Council, Making the Nation Safer: The Role of Science and Technology in Countering Terrorism, National Academies, Washington, DC, 2002.

[4] D.L. Chichester, J.D. Simpson, Compact accelerator neutron generators, The Industrial Physicist, December 2003-January 2004, pp. 22-25.

[5] (a) For example: J.D. Gow, H.C. Pollock, Review of Scientific Instruments 31 (1960) 235;

(b) L.T. Perkins, C. Celata, Y. Lee, K.N. Leung, D.S. Picard, R. Vilaithong, M.D. Williams, D. Wutte, AIP Conference Proceedings 392 (1997) 1191;

(c) K. Nishimura, M. Kato, Y. Miake, Y. Rintsu, Fusion Technology 39 (2001) 1174

(d) J. Reijonen, F. Gicquel, S.K. Hahto, M. King, T.P. Lou, K.N. Leung, Applied Radiation and Isotopes 63 (2005) 757;

(e) L. Rapezzi, M. Angelone, M. Pillon, E. Rossi, M. Samuelli, F. Mezzetti, Plasma Sources Science \& Technology 13 (2004) 272.

[6] P.R. Schwoebel, Applied Physics Letters 87 (2005) 054104

[7] P.R. Schwoebel, J. Brodie, Journal of Vacuum Science \& Technology B 13 (1995) 1391.

[8] I. Solano, B. Reichenbach, P.R. Schwoebel, D.L. Chichester, C.E. Holland, K.L. Hertz, J.P. Brainard, Nuclear Instruments \& Methods in Physics Research A $11(2008) 76$

[9] B. Reichenbach, High Electric Field Deuterium Ion Sources for Neutron Generators, Ph.D. Thesis, University of New Mexico, <http://hdl.handle.net/ $1928 / 10332>$, September 2009.

[10] R. Gomer, Surface Science 299 (1994) 129.

[11] J.A. Panitz, Progress in Surface Science 8 (1978) 219

[12] Supplied by: Sandia National Laboratories, Albuquerque, NM.

[13] Supplied by: Sandia National Laboratories, Livermore, CA.

[14] B. Reichenbach, I. Solano, P.R. Schwoebel, Journal of Applied Physics 103 (2008) 094912.

[15] B. Reichenback, B.Bargsten Johnson, Journal of Applied Physics 108 (2010) 094903.

[16] R. Gomer, Field Emission and Field Ionization, Harvard University Press Cambridge, 1961 (132 pp.).

[17] E. Krautz, M. Leisch, Mikrochimica Acta 74 (1980) 435.

[18] D.L. Chichester, J.P. Brainard, P.R. Schwoebel, K.L. Hertz, C.E. Holland, Nuclear Instruments \& Methods in Physics Research B 261 (2007) 835.

[19] To be published, K.L. Hertz.

[20] P.J. Resnick, C.E. Holland, P.R. Schwoebel, K.L. Hertz, D.L. Chichester, Microelectronic Engineering 87 (2010) 1263.

[21] W. Zhu, Vacuum Microelectronics, John Wiley \& Sons, New York, 2001 (105 pp.).

[22] H.A.M. Van Eekelen, Surface Science 21 (1970) 21.

[23] G.R. Hanson, Journal of Chemical Physics 62 (1975) 1161

[24] (a) For example: A. Jason, B. Halpern, M.G. Inghram, R. Gomer, Journal of Chemical Physics 52 (1970) 2227;

(b) A. Jason, R.P. Burns, M.G. Inghram, Journal of Chemical Physics 43 (1965) 3763;

(c) G.R. Hanson, Microelectronics: Applications, Materials and Technology 2 (1984) 17.

[25] L.A. Shope, Sandia National Laboratories Report S.C.-TM-66-247, 1966.

[26] E.W. Muller, T.T. Tsong, Progress in Surface Science 4 (1974) 1. 